\title{
Detox - Haemofiltration for The Neuroleptic Malignant Syndrome
}

\author{
Juan F Martin Lazaro'*, Marc Brown ${ }^{1}$, Emily Gould ${ }^{1}$, Johann Grundlingh ${ }^{1}$, Michal Brys Ieva Norkiene ${ }^{1}$, Joaquim \\ Cevallos $^{1}$, James Napier ${ }^{1}$, Khyati Lad ${ }^{2}$ and Jacek Jablonski ${ }^{3}$
}

${ }^{1}$ Intensive Care Unit. Newham University Hospital, UK

${ }^{2}$ Pharmacy Department, Newham University Hospital, UK.

${ }^{3}$ Beloit Memorial Hospital, USA

*Corresponding author: Juan F Martin Lazaro, Intensive Care Unit, Newham University Hospital, London, UK

\begin{abstract}
Removal of serum toxins using haemofiltration with human albumin has been demonstrated to be a useful option for the treatment of a patient following the ingestion of unknown substance. Here, a case is described in which a patient presented with symptoms of neuroleptic malignant syndrome after ingesting an unknown Nigerian herbal remedy. Albumin enhanced continuous veno-venous haemodialysis has been previously used for intoxications. When combining continuous renal replacement therapy with albumin, the toxins are bound to the larger molecule which prevents the toxins moving through the filter; allowing for clearance from the blood. We suggest that Intensive Care Units should consider basic tools, such as detox haemofiltration when an unknown poison is suspected.
\end{abstract}

\section{Introduction}

Neuroleptic malignant syndrome (NMS) is a medical emergency, made more difficult by the lack of any diagnostic investigations, making it an entirely clinical diagnosis. Four criteria are used as parameters for a likely diagnosis: a change in mental status, a muscle rigidity (lead-pipe type), hyperthermia and signs of autonomic instability. TOXBASE guidelines state that in cases of suspected poisoning, activated charcoal may be used within 1 hour of ingestion; However, in cases like this where the patient is late in presentation, options are more limited. To our current knowledge, there is no specific antidote, and recommended therapy is supportive only. A few case reports have suggested the use of CVVH, peritoneal dialysis, and haemodialysis. In the absence of any diagnostic tests, we felt it was prudent to initiate renal replacement therapy in an attempt to filter any precipitating substances, without waiting for the development of further symptoms. Here we discuss the use of albumin enhanced continuous renal replacement therapy to treat a likely neuroleptic malignant syndrome.

\section{Case Report}

A 60-year-old male presented to the Accident and Emergency Department with reduced conscious level, diarrhoea and vomiting. The patient had ingested an unknown powder sent from Nigeria two days before presentation, for use as a treatment for his recently diagnosed Hepatitis B infection. Other past medical history includes type 2 diabetes mellitus, hypertension and hypercholesterolemia, treated with metformin, gliclazide, ramipril and atorvastatin respectively. The patient's wife subsequently gave a collateral history, explaining that the patient had taken the unknown substance from Nigeria, knowing that it would make him very unwell for 3 days. She called for the ambulance on the $2^{\text {nd }}$ day due to his reduced conscious level and vomiting. Upon initial assessment, respiratory rate was $35 / \mathrm{min}$, heart rate $115 / \mathrm{min}$, blood pressure $100 / 60 \mathrm{mHg}$ and temperature of $390 \mathrm{c}$. On auscultation he had crackles at both lung bases, a finding consistent with a chest $x$ ray that demonstrated bilateral infiltrates, suggestive of either bronchoaspiration or 
pulmonary oedema. Cardiovascular and abdominal examinations were otherwise unremarkable. On neurological examination, there was severe lead pipe rigidity of all four limbs and neck stiffness accompanying a Glasgow Coma Score (GCS) of $10\left(E_{3} V_{2} M_{5}\right)$, though with normal pupillary reflexes. Arterial blood gas results were: $\mathrm{pH}$ $7.22 \mathrm{pCO}_{2}$ 7.11, $\mathrm{pO}_{2}$ 14.4, $\mathrm{Hb}$ 132, Glucose 12.6, Lactate 1.6, Base Excess- 4.9, $\mathrm{HCO}_{3}$ 19.4. Abnormal blood tests included: Urea 15.8, Creatinine 207, CK 1600, WCC 3.1 (neutrophils 2.1, lymphocytes 0.8), INR 1.4. CT Thorax abdomen and pelvis showed bilateral patchy areas of consolidation, ground-glass opacities and air space opacities suggestive of aspiration pneumonia. CT head was normal. [1-4] ECG and bedside echocardiography were normal. An uncomplicated intubation was performed due to hypercapnic respiratory failure and reduced GCS, and the patient was transferred to the intensive care unit. A nasogastric tube was inserted and $300 \mathrm{ml}$ of yellow-brown fluid was removed. The following antibiotic treatment was commenced: co-amoxiclav, clarithromycin and amikacin. High flow continuous veno-venous haemofiltration in combination with IV human albumin was started to remove potential precipitating agent. The patient improved quickly, and the small dose of noradrenaline started on day 1 was weaned off within 24 hours. The muscle rigidity improved slowly over 4 days but became the primary issue, along with inappropriate waking from sedation. A repeat CT head was performed which was normal, and an EEG was also normal. The neurological symptoms improved by day 5 and he was successfully extubated. He spent a subsequent 3 days on the unit until his pneumonia was fully resolved and was discharged on day 9.

\section{Methods}

High volume veno-venous filtration was performed using an Aquarius System. Blood was driven through a highly permeable haemofilter type Aquamax HF 12 poly-ether-sulfone from Baxter (cut-off point of 30.000 Dalton); systemic anticoagulation was also added into the haemofilter and pre-dilution mode was used. Extracorporeal blood flow ranged from 250 to $360 \mathrm{ml} / \mathrm{min}(60 \mathrm{ml} /$ $\mathrm{Kg}$ ). The principle of clearance in this model of continuous dialysis was convection. No fluid was taken from this patient to maintain haemodynamic stability. The procedure was performed over the course of $48 \mathrm{~h}$. Albumin 20\%,100ml, was administered three times during the procedure due to the expectation that toxins would bind to the albumin which has a high molecular weight and will be trapped by the filter .Toxicology was contacted on admission; Dantrolene was considered but not administered, as it was unclear at the time whether this was a NMS; we were advised to use it only if autonomic disturbances were present. A screening of $>1000$ substances were tested in the National Laboratory of Toxicology but non potential toxics were identified.

\section{Discussion}

According to the patient's family, the substance which he ingested was a soluble powder sent from Nigeria called "agumu".
After researching this term, we have discovered this is a generic name for "medicine" and has multiple different references, but not 1 specific treatment [5-8]. The substance was sent for toxicological analysis at a national laboratory testing for 1500 different drugs but was not flagged as positive for any of them. We performed a literature search and found that there is a very limited amount of information regarding Nigerian herbal remedies which fit the description provided by the family of the patient. We were able to identify 1 compound, Rauwolfia Vomitoria that has been used in Nigerian herbal remedies for several purposes, and is known for its anticonvulsant, analgesic and antipsychotic properties. This compound has a similar effect profile as Chlorpromazine, which is a dopamine antagonist, and can cause neuroleptic malignant syndrome in overdose. Unfortunately, we did not discover the true identity of the substance, so further speculation is not helpful. However, the usefulness of the albumin haemofiltration may still be discussed. Many antipsychotic medications are highly lipid soluble and have high levels of protein binding. The above example of chlorpromazine has a $90-99 \%$ protein binding capacity. In the severely affected patients, it is theoretical that increasing serum albumin levels would increase the bound fraction of the drug, and therefore prevent the drug from moving through the filter. On the other hand, it is not clear whether patients with a normal endogenous serum albumin concentration would already have sufficient levels of protein binding capacity. Due to the severity of the case, the potential harm caused by line insertion and filtration is likely outweighed by the potential benefit, as these patients often end up on the filter due to renal failure secondary to either direct drug nephrotoxicity or secondary effects from high creatinine kinase levels.

\section{Conclusion}

Thankfully, this patient made a full recovery, and we have counselled him regarding the ingestion of unknown substances before discussion with a doctor. Due to many confounding factors in this case, it is difficult to demonstrate causality with any single intervention. However, these patients can prove to be challenging, especially in the early stages of presentation where the history is unclear. With the lack of diagnostic tests, and the inevitable delay in serum drug sampling, it is reasonable to start therapies which are likely to do little harm but may have a large positive impact. We believe the albumin hemofiltration is such an example.

\section{References}

1. Askenazi DJ, Goldstein SL, Chang IF, Elenberg E, Feig DI (2004) Management of a severe carbamazepine overdose using albuminenhanced continuous venovenous hemodialysis. Pediatrics 113(2): 406409.

2. Velamoor VR, Norman RM, Caroff SN (1994) Progression of symptoms in neuroleptic malignant syndrome. J Nerv Ment Dis 182: 168-73.

3. Frucht SJ (2014) Treatment of movement disorder emergencies. Neurotherapeutics 11(1): 208-212.

4. Van der Vusse GJ (2009) Albumin as fatty acid transporter. Drug Metab Pharmacokinet 24(4): 300-307. 
5. Martin Lazaro JF, Janjanin S, Kulhalli V, Napier J (2017) Conventional High-Volume Hemofiltration for Hypertriglyceridemic Pancreatitis. Journal of Clinical Nephrology and Renal Care 3: 020.

6. M Eluwa, N Idumesaro, M Ekong, An Akpantah (2008) T Ekanem Effect of Aqueous Extract of Rauwolfia Vomitoria Root Bark on The Cytoarchitecture Of the Cerebellum and Neurobehaviour Of Adult Male Wistar Rats. The Internet Journal of Alternative Medicine 6(2).
7. Aminoff MJ (2004) Pharmacologic management of parkinsonism and other movement disorders. In: Katzung BG, editor. Basic Clinical Pharmacology. Singapore: McGraw-Hill, pp. 447-461.

8. Amole 00, Yemitan OK, Oshikoya KA (2009) Anticonvulsant activity of Rauwolfia vomitoria (Afzel) African Journal of Pharmacy and Pharmacology 3: 319-322.

\section{(c) (P) \\ This work is licensed under Creative Commons Attribution 4.0 License}

To Submit Your Article Click Here:

Submit Article

DOI: $10.32474 /$ GJAPM.2019.01.000111

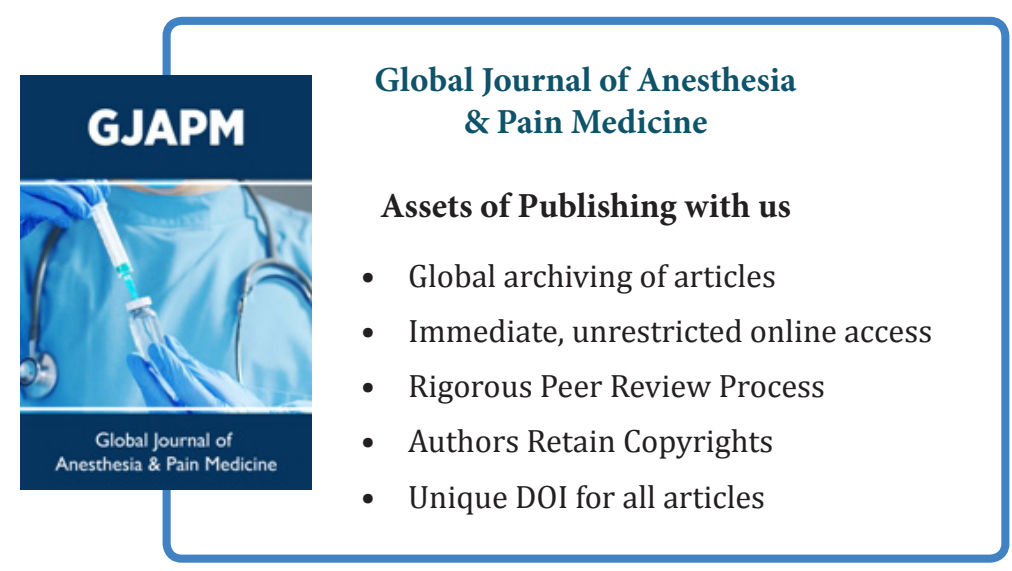

